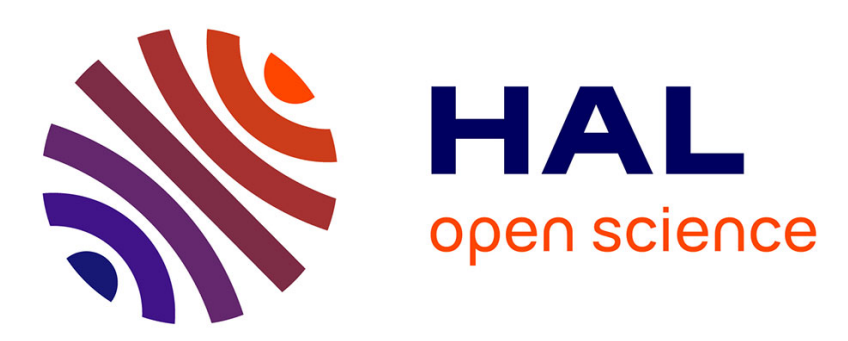

\title{
Control over the Electrostatic Self-assembly of Nanoparticle Semiflexible Biopolyelectrolyte Complexes
}

Li Shi, Florent Carn, François Boué, Gervaise Mosser, Eric Buhler

\section{To cite this version:}

Li Shi, Florent Carn, François Boué, Gervaise Mosser, Eric Buhler. Control over the Electrostatic Selfassembly of Nanoparticle Semiflexible Biopolyelectrolyte Complexes. Soft Matter, 2013, 9, pp.50045015. 10.1039/C3SM27138B . hal-00803042

\section{HAL Id: hal-00803042 \\ https://hal.science/hal-00803042}

Submitted on 20 Mar 2013

HAL is a multi-disciplinary open access archive for the deposit and dissemination of scientific research documents, whether they are published or not. The documents may come from teaching and research institutions in France or abroad, or from public or private research centers.
L'archive ouverte pluridisciplinaire HAL, est destinée au dépôt et à la diffusion de documents scientifiques de niveau recherche, publiés ou non, émanant des établissements d'enseignement et de recherche français ou étrangers, des laboratoires publics ou privés. 


\title{
Control over the Electrostatic Self-assembly of Nanoparticle Semiflexible Biopolyelectrolyte Complexes $\uparrow$
}

\author{
Li Shi, ${ }^{\text {a,b }}$ Florent Carn, ${ }^{\text {a }}$ François Boué, ${ }^{\mathrm{b}}$ Gervaise Mosser ${ }^{\mathrm{c}}$ and Eric Buhler ${ }^{\text {*a,b }}$ \\ Received (in $X X X, X X X)$ Xth $X X X X X X X X X 20 X X$, Accepted $X$ th $X X X X X X X X X 20 X X$ \\ ${ }_{5}$ DOI: 10.1039/b000000x
}

The electrostatic complexation between model negatively charged silica nanoparticles (NPs) with radius $\mathrm{R} \sim 10 \mathrm{~nm}$ and chitosan, a natural polyelectrolyte bearing positive charges with a semi-rigid backbone of persistence length of $\mathrm{L}_{\mathrm{p}} \sim 9 \mathrm{~nm}$, was studied by a combination of SANS, SAXS, light scattering, and cryoTEM. In this system, corresponding to $\mathrm{L}_{\mathrm{p}} / \mathrm{R} \sim 1$, we observe the formation of (i) randomly branched

10 complexes in the presence of an excess of chitosan chains and (ii) well-defined single-strand nanorods in the presence of an excess of nanoparticles. We also observe no formation of nanorods for NPs with polyL-lysine, a flexible polyelectrolyte, corresponding to $\mathrm{L}_{\mathrm{p}} / \mathrm{R} \sim 0.1$, suggesting a key role played by this ratio $\mathrm{L}_{\mathrm{p}} / \mathrm{R}$. In the intermediate range of nanoparticles concentrations, we observe an associative phase separation (complex coacervation) leading to more compact complexes in both supernatant and

15 coacervate phases. This method might open the door to a greater degree of control of nanoparticles selfassembly into larger nanostructures, through molecular structural parameters like $\mathrm{L}_{\mathrm{p}} / \mathrm{R}$, combined with polyelectrolytes/nanoparticles ratio.

\section{Introduction}

The spatial orientation and arrangement of nanoparticles (NPs) is 20 important in the realization of technologically useful nanoparticle-based materials ${ }^{1-4}$ and today the focus of nanotechnology is gradually shifting from the synthesis of individual NPs to the self-organization of larger nanostructures. ${ }^{5}$ The physical and chemical properties of ensembles of NPs are 25 strongly dependent on their shape as well as their size (R) and are determined by collective interactions of individual NPs, ${ }^{6}$ generating nanostructures with complex hierarchical architectures. $^{7-15}$ For instance the establishment of a useful and controllable methodology for the assembly of NPs into well30 defined rods or arrays is peculiarly important because it offers immense opportunities of applications in optoelectronics, ${ }^{16-20}$ micro imaging, sensing, ${ }^{21,22}$ therapeutics, etc. In spite of this potential, the assembly of NPs into well-defined nanorods or nanowires remains a challenge. Previously, one-dimensional (1D)

35 nanostructures have been prepared by several ways, such as photo-reduction using UV irradiation, ${ }^{23}$ template-directing using membrane ${ }^{24}$ or mesoporous silica, ${ }^{25}$ solution-phase hydrothermalreduction using various polymer surfactants, ${ }^{26-30}$ or using proteins as templates. ${ }^{1}$ During the last decade several research groups 40 reported on the assembly of colloidal particles in 1D polymer-like structures using various sophisticated chemical and physical processes. $^{9,14,15,31-35}$ Also a reaction-controlled step-growth polymerization of inorganic functionalized NPs has been reported very recently. ${ }^{36,37}$ But in the landscape of these different 45 advanced approaches, appears the need to develop less sophisticated and cost-efficient methodologies that can be used to construct nanostructures with desired size and shape.
This is the case with the route studied in this paper: the use of 50 electrostatic interaction, a simple process with interesting potentialities. Association between different species of opposite charge can lead to new objects, often called electrostatic complexes. A case widely studied recently is complexes formed by electrostatic interaction between polyelectrolytes (PEL) and 55 NPs of opposite charge. ${ }^{38-41}$ Theoretical and experimental works have revealed that along with the physico-chemical parameters such as charge concentration ratio, $\mathrm{pH}$ and ionic strength acting on all ionizable species, the characteristics of polymer systems yield additional original tuning parameters. ${ }^{42-44}$ In the dilute 60 regime, most of the studies focused on the local interaction between a short segment of the polymer chain and one oppositely charged NP. Parameters involving larger scales, like the total polyelectrolyte length or the number of NPs per chain have been taken into account only by simulation. ${ }^{45}$ At intermediate scales, ${ }_{65}$ experiments are also missing on the influence of NPs surface charge density, shape, and size, as well as of the persistence length $\mathrm{L}_{\mathrm{p}}$, a rigidity parameter specific to polymers, the influence of which has been studied theoretically and by simulations. ${ }^{42-44}$ The case of DNA however, due to its biophysical interest, was 70 more studied, like ten years ago by Keren et al. using doublestrand DNA and charged gold NPs. ${ }^{46}$ The authors revealed the existence of DNA/AuNPs soluble complexes and discussed the stability of the solutions as a function of the polyelectrolyte/nanoparticle concentration ratio. Interestingly, 75 their soluble complexes seem to adopt a kind of "bead on a string" shape observed by AFM, SEM and TEM after deposition on "sticky" silicon wafer. However, the shape of the complexes 
has not been studied in bulk. Also these interesting effects were observed only for DNA, which is a rigid polymer $\left(\mathrm{L}_{\mathrm{p}} \geq 50 \mathrm{~nm}\right)$ while for many polyelectrolytes $\mathrm{L}_{\mathrm{p}}$ ranges between 10 and $1 \mathrm{~nm}$ and for one NP size ( $\mathrm{R}=8 \mathrm{~nm})$ only. Our approach is to study the 5 complex structure for different $\mathrm{L}_{\mathrm{p}}$ and different sizes so that the influence of the ratio $\mathrm{L}_{\mathrm{p}} / \mathrm{R}$ can be established. Also the structure will be studied in situ in bulk, without influence of a surface.

The NPs we have chosen are not gold but silica synthetic NPs with radius 10nm: they are generally considered as 10 very simple model systems, with fixed characteristics (size, shape, and surface function), regular shape and homogeneous surface. Under well-defined conditions a sensible evaluation of the different kinds of interactions (electrostatic, hydrogen bonds, hydrophobic) can be made showing that electrostatic interactions 15 dominate and can be estimated - contrary to proteins, ${ }^{47-53}$ which are more complex. SiNPs usually bear negative charges, so that they can be combined with species of opposed charges. The case of positive compact particles, namely lysozyme proteins ${ }^{54}$ (smaller than SiNPs), in interaction with SiNPs has already been 20 studied, showing nicely for example that interaction occurs when the two global charges are of opposite charge, a point also often discussed by Dubin's group. ${ }^{48,49}$ But our study focus here on the case where the oppositely charged species is a polyelectrolyte (PEL). We have chosen chitosan, a well-known polysaccharide 25 polyelectrolyte bearing positive charges and displaying a semiflexible backbone characterized by a persistence length of $\mathrm{L}_{\mathrm{p}} \sim 7.5$ $\mathrm{nm},{ }^{55,56}$ In our system, electrostatic complexation takes place in a narrow range of $\mathrm{pH}$, which we therefore kept at 4.5 , but it is also well defined. Chitosan chain wrapping around the NPs (not 30 possible with positive dense objects like proteins) is used to induce well-defined shapes. The phase diagram as a function of chitosan and SiNP concentrations at fixed charge density enables to characterize soluble "single complexes" in excess of SiNPs or of chitosan, and a region of coexistence of poor/rich phase 35 (coacervate). We have in particular revealed for $\mathrm{L}_{\mathrm{p}} / \mathrm{R} \sim 1$ the presence of well-defined single physical complexes displaying a 1D array structure in excess of NPs and an elongated branched structure in excess of chitosan. For comparison, we also show briefly here that poly-L-lysine, a flexible polyelectrolyte, with $\mathrm{L}_{\mathrm{p}}$ $40=1 \mathrm{~nm}\left(\mathrm{~L}_{\mathrm{p}} / \mathrm{R} \sim 0.1\right)$, does not induce the formation of $1 \mathrm{D}$ structures. These results highlight the major role played by the polyelectrolyte persistence length.

Here, we study the structure of what we call "single complex", the physical assembly of NPs complexed with a small 45 number of oppositely charged polymer chains. The single complex is expected to be of nanometric size and to be present in the monophasic or diphasic systems. Thanks to the convenient suite of small-angle X-ray and neutron scattering q-range combined with the light scattering one, we have fully 50 characterized the structure of these objects: mass, radius, linear density. Molecular structural parameters like the ratio $\mathrm{L}_{\mathrm{p}} / \mathrm{R}$ and phase diagram determination enable a quantitative prediction of their structural features: shape, size, NPs organization, number of NPs in an ensemble, formation 1D arrays. This methodology

55 might open the door to a greater degree of control over the way NPs assemble into larger nanostructures.

In brief, our study brings new original views: (i) this "electrostatic route" is simple, and we show for the first time that it can lead, in particular, to rods made out of nanoparticles with 60 monodisperse cross-section. The mechanism is original, different from former cases such as Au/DNA: the rods are formed from chitosan chains of persistence length lower than $10 \mathrm{~nm}$, i.e., 25 times shorter than the nanorods (meaning a strong tightening of the polymer chain), and 7 times less rigid than DNA. (ii) We
65 observe experimentally for the first time a structural change in two very similar systems where the main difference is the characteristic ratio $\mathrm{L}_{\mathrm{p}} / \mathrm{R}$. This makes plausible a cognitive generalization to many other candidate species. (iii) Together with simplicity, the process is robust, fast, green, low cost, and it 70 should be applied to metallic NPs that have been attracting many concerns for their remarkable industrial potential.

\section{Experimental section}

\section{Sample Characteristics}

75 Chitosan. Polysaccharide chitosan belongs to a family of linear cationic biopolymers obtained from alkaline $\mathrm{N}$ deacetylation of chitin, which is the second most abundant polymer in nature. The chitosan studied here is a commercial polymer (with polydispersity index around 1.3) from Sigma${ }_{80}$ Aldrich composed of $\beta \quad 1 \rightarrow 4$ D-glucosamine units with a degree of $\mathrm{N}$-acetylation equal to $12.5 \%$ (determined by NMR). The mass and the length of the monomer are respectively equal to 166 $\mathrm{g} / \mathrm{mol}$ and $5 \AA$. In acid conditions, chitosan is water-soluble due to the presence of protonated amino groups. The solutions were then 85 investigated in the solvent $0.3 \mathrm{M}$ acetic acid $\left(\mathrm{CH}_{3} \mathrm{COOH}\right)$ in the presence of $0.2 \mathrm{M}$ sodium acetate $\left(\mathrm{CH}_{3} \mathrm{COONa}\right)$. We obtain thus a $\mathrm{pH}=4.5$ buffer where all the amino groups bear a positively charged proton. So chitosan exhibits a high polyelectrolyte character with one positive charge every $5 \AA$ A ${ }^{55-58}$ which would be 90 reduced to one charge per $7 \AA$ after Manning condensation. The intrinsic persistence length of chitosan backbone is roughly equal to $7.5 \mathrm{~nm},{ }^{55}$ which ranges it in the class of the so-called semirigid polyelectrolytes. The weight-average molecular weight, $M_{\mathrm{W}}=313 \pm 20 \mathrm{~kg} / \mathrm{mol}$, was determined using static light scattering 95 measurements.

Poly-L-lysine (PLL). PLL (chemical formula $\left(\mathrm{C}_{6} \mathrm{H}_{12} \mathrm{~N}_{2} \mathrm{O}\right)_{\mathrm{n}}$, monomer mass is equal to $\left.128 \mathrm{~g} / \mathrm{mol}\right)$ is a natural homopolymer composed of L-lysine amino acids and produced 100 by bacterial fermentation. Each unit of the chain contains an amino group $\left(\mathrm{NH}_{3}{ }^{+}\right)$that renders the whole chain positively charged with a $\mathrm{pKa}=9$. Here, PLL was chosen because it has both positive charges, to interact with negative SiNPs, and a flexible backbone characterized by a persistence length close to $1 \mathrm{~nm}$, 105 much shorter than the one of chitosan. The poly-L-lysine hydrobromide used in our study was purchased from SigmaAldrich in powder state and was used as purchased. Aqueous solutions were prepared in the presence of $0.2 \mathrm{M}$ of $\mathrm{KBr}$. The concentration of additional salt $\mathrm{KBr}$ was fixed at $0.2 \mathrm{M}$ to keep 110 the same ionic strength than that of chitosan solutions. Within these experimental conditions, all the amino groups are protonated and PLL is fully charged displaying a charge approximately every $3.5 \AA$ (monomer size); i.e., every $7 \AA$ after Manning correction. The molar weight of PLL was determined by 115 static light scattering (SLS) using a classical Zimm analysis. One obtains $\mathrm{M}_{\mathrm{W}}=54000 \mathrm{~g} / \mathrm{mol}$ and $\mathrm{R}_{\mathrm{G}}=4.6 \mathrm{~nm}$.

Silica NPs. Silica dispersions of the desired concentration were obtained by dilution of the required quantity of commercial 120 dispersion ( $\left[\mathrm{SiO}_{2}\right]=30$ wt.\%) of Ludox $\mathrm{AM}$ in the $0.3 \mathrm{M}$ $\mathrm{CH}_{3} \mathrm{COOH} / 0.2 \mathrm{M} \mathrm{CH}_{3} \mathrm{COONa}$ buffer of $\mathrm{pH} 4$.5. In Ludox AM, tetravalent silicon ions have been substituted for part by trivalent aluminium ions $\left(\left[\mathrm{Al}_{2} \mathrm{O}_{3}\right]=0.2 \mathrm{wt}\right.$.\% in our dispersion according to the supplier) on the surface of the particles. Therefore, these 
modified silica particles carry a more pronounced negative surface charge density over a wide $\mathrm{pH}$ range giving rise to very good stability against variation of $\mathrm{pH}$ (see ESI). ${ }^{59-61}$ Such stability with time of the SiNPs solutions has been checked using light 5 scattering measurements, see ESI. ${ }^{62-66}$

\section{Small-Angle Neutron Scattering Experiments (SANS)}

SANS experiments were carried out using PACE (Léon Brillouin Laboratory-LLB at Saclay, France) and D11 (Institut Laue ${ }_{10}$ Langevin-ILL, Grenoble) spectrometers. The chosen incident wavelength, $\lambda$, depends on the set of experiments, as follows. For a given wavelength, the range of the amplitude of the transfer wave vector $q$ was selected by changing the detector distance, D. Two sets of sample-to-detector distances and wavelengths were 15 chosen at $\operatorname{LLB}(D=1.0 \mathrm{~m}, \lambda=10 \pm 1.0 \AA$; and $D=4.7 \mathrm{~m}, \lambda=10$ $\pm 1.0 \AA)$ so that the following $q$-ranges were respectively available: $2.2 \times 10^{-2} \leq q\left(\AA^{-1}\right) \leq 2.2 \times 10^{-1}$, and $4.2 \times 10^{-3} \leq q\left(\AA^{-1}\right) \leq$ $4.4 \times 10^{-2}$. At ILL (dilute simple solution of chitosan) we used $D=$ $8 \mathrm{~m}$ and $\lambda=5 \pm 0.5 \AA$, giving a $q$-range of $8.4 \times 10^{-3} \leq q\left(\AA^{-1}\right) \leq$ 200.104 . Measured intensities were calibrated to absolute values $\left(\mathrm{cm}^{-1}\right)$ using normalization by the attenuated direct beam classical method. Standard procedures to correct the data for the transmission, detector efficiency, and backgrounds (solvent, empty cell, electronic, and neutronic background) were carried 25 out.

The usual equation for absolute neutron scattering intensity combines the intraparticle scattering $S_{1}(q)=V_{\text {chain }} \phi_{v o l} P(q)$ factor $\left(\mathrm{P}(\mathrm{q})\right.$ is the form factor) with the interparticle scattering $\mathrm{S}_{2}(\mathrm{q})$ factor

${ }_{30} I(q)\left(\mathrm{cm}^{-1}\right)=(\Delta \rho)^{2}\left(S_{1}(q)+S_{2}(q)\right)=(\Delta \rho)^{2}\left(V_{\text {chain }} \phi_{\text {vol }} P(q)+S_{2}(q)\right)$

where $(\Delta \rho)^{2}=\left(\rho_{\text {monomer }}-\rho_{\text {solvent }}\right)^{2}$ is a contrast per unit volume between the polymer and the solvent, which was determined from the known chemical composition. $\rho=\Sigma \mathrm{n}_{\mathrm{i}} b_{\mathrm{i}} /\left(\sum \mathrm{n}_{\mathrm{i}} m_{\mathrm{i}} v \times 1.66 \times 10^{-24}\right)$ 35 is the scattering length density per unit volume (SLDs), $b_{\mathrm{i}}$ is the neutron scattering length of the species $\mathrm{i}, m_{\mathrm{i}}$ the mass of species $\mathrm{i}$, and $v$ the specific volume of the monomer (which was measured and taken equal to 0.478 and $0.4545 \mathrm{~cm}^{3} \mathrm{~g}^{-1}$ for chitosan ${ }^{67}$ and silica, respectively) or the solvent (i.e., $0.9058 \mathrm{~cm}^{3} \mathrm{~g}^{-1}$ for 40 deuterated water). $V_{\text {chain }}=\mathrm{N} v m \times 1.66 \times 10^{-24}$ is the volume of the $\mathrm{N}$ monomers (of mass $m$ ) in a chain and $\phi_{\mathrm{vol}}$ is the volume fraction of monomer. In the high $q$-range, the scattering is assumed to arise from isolated chains; i.e., $\mathrm{S}_{2}(q)=0$, and thus $\mathrm{I}(q) \propto \mathrm{P}(q)$.

\section{${ }_{45}$ Small-Angle X-Ray Scattering Experiments (SAXS)}

The SAXS experiments were performed at the ESRF (Grenoble, France) on the ID-02 instrument using the pinhole camera at the energy of $12.46 \mathrm{keV}$ at two sample-to-detector distances $(1 \mathrm{~m}$ and $8 \mathrm{~m})$ corresponding to a q-range varying between $0.0011 \AA^{-1}$ and ${ }_{50} 0.57 \AA^{-1}$. The absolute units are obtained by normalization with respect to water (high q-range) or lupolen (low q-range) standard. For SAXS, the scattering length densities (SLDs) are defined by $\rho=1 /\left(\operatorname{mv} \times 1.66 \times 10^{-24}\right) \times r_{e l} \times \Sigma n_{i} Z_{i}$, where $r_{e l}=0.28 \times 10^{-5} \mathrm{~nm}$ is the electron radius and $Z_{i}$ the atomic number of element $i$. Table 1 in ${ }_{55}$ ESI reports the scattering length densities SLDs per unit volume of chitosan and silica calculated for SANS and SAXS.

The polydispersity in size of the scattered objects have been described by a log-normal distribution, $\mathrm{L}(\mathrm{r}, \mathrm{R}, \sigma)$, where $\mathrm{r}$ is the ${ }_{60}$ radius, $\mathrm{R}$ the mean radius, and $\sigma$ the variance:

$$
L(r, R, \sigma)=\frac{1}{r \sigma \sqrt{2 \pi}} \exp \left(-\frac{1}{2 \sigma^{2}} \ln ^{2}\left(\frac{r}{R}\right)\right)
$$

Thus, neglecting the Virial effects (neglecting interparticles correlations), it is classical to define the global scattering intensity by the following relation: ${ }^{60}$

${ }^{65} I(q)=\phi(\Delta \rho)^{2} V \int_{0}^{\infty} P(q, r) L(r, R, \sigma) d r$

$\mathrm{q}=4 \pi / \lambda \times \sin \theta / 2$ is the wave vector, $(\Delta \rho)^{2}$ the contrast factor, $\phi$ the volume fraction, $\mathrm{V}$ the volume of the scattered objects, and $\mathrm{P}(\mathrm{q}, \mathrm{r})$ the form factor. Although silica and chitosan SLDs are close for both neutron and X-rays, the signal is dominated by the scattering 70 of the SiNPs and the signal of chitosan chains is negligible due to the high volume and compacity of the SiNPs.

\section{Cryogenic Transmission Electron Microscopy (Cryo-TEM)}

Cryo-transmission electron microscopy (cryo-TEM) was 75 performed on vitrified complexes prepared at several chitosan and NPs concentrations characterizing the different domains of the phase diagram. In brief, a $5 \mathrm{ml}$ drop of the solution to be imaged was set onto a TEM QUANTIFOIL carbon coated grid. The drop was blotted with Whatman filter paper \#4, and the grid 80 was quenched rapidly in liquid ethane to avoid the crystallization of the aqueous phase. The vitrified samples were then stored under liquid nitrogen until their transfer onto a Gatan cryo-holder operating at $-180^{\circ}$. Samples were analysed on a FEI Tecnai Spirit G2 TEM microscope operating at $120 \mathrm{kV}$. Images were recorded 85 on a Gatan Orius CCD camera at $40000 \times$.

\section{Results and Discussion}

\section{Single solute solutions characterization}

In this paragraph, we briefly summarize the characterization performed on each component (chitosan and SiNPs) before 90 mixing to ascertain their dimensions and initial dispersion state in the solvent used. The full characterization obtained using SAXS, static and dynamic light scattering is presented in the ESI.

The buffer solution is the aqueous solution consisting of a 95 mixture of $0.3 \mathrm{M} \mathrm{CH}_{3} \mathrm{COOH}$ and $0.2 \mathrm{M} \mathrm{CH}_{3} \mathrm{COONa}$. Since, as said above, SiNPS and chitosan chains are both fully charged in a relatively small $\mathrm{pH}$ range, this buffer was used as a mean of keeping the $\mathrm{pH}$ at a constant value of 4.5. The solvent is a good solvent for both components and the electrostatic complexation 100 between the two partners takes place readily. We did not vary either the ionic strength. Table 1 summarizes the structural characteristics of the two partners. 


\begin{tabular}{|l|l|}
\hline Chitosan & SiNPs \\
\hline $\mathrm{R}_{\mathrm{G}}=66 \pm 5 \mathrm{~nm}$ & $\mathrm{R}=9.2 \mathrm{~nm}$, variance $=0.12$ \\
$\mathrm{R}_{\mathrm{H}}=44 \pm 4 \mathrm{~nm}$ & $\mathrm{R}_{\mathrm{H}}=11.7 \pm 1.5 \mathrm{~nm}$ \\
$\mathrm{M}_{\mathrm{W}}=313 \pm 20 \mathrm{kDa}$ & $\mathrm{M}_{\mathrm{W}}=(3 \pm 0.2) \times 10^{6} \mathrm{Da}$ \\
$\mathrm{L}_{\mathrm{c}}=943 \mathrm{~nm}$ & \\
\hline
\end{tabular}

Table 1 Characteristics of the two partners determined using light scattering and SAXS experiments: radius of gyration, $\mathrm{R}_{\mathrm{G}}$, radius, $\mathrm{R}$, hydrodynamic radius, $\mathrm{R}_{\mathrm{H}}$, weight-average molecular weight, ${ }_{5} \mathrm{M}_{\mathrm{W}}$, and contour length, $\mathrm{L}_{\mathrm{c}}$ (see ESI for details).

An important point to be taken care of before dealing with the phase behavior of the mixtures is the precise determination of chitosan persistence length, a parameter playing a major role in 10 complexation phenomena. Figure 1 displays the scattering pattern of a $0.1 \mathrm{~g} / \mathrm{l}$ chitosan solution obtained by coupling SLS (low-q data) and SANS measurements. We normalized, for both techniques, the scattered intensity by the corresponding contrast term. Then assuming that $0.1 \mathrm{~g} / \mathrm{l}$ is a concentration low enough to 15 consider the solution as dilute, the result is $\mathrm{M}_{\mathrm{W} \times} \mathrm{P}(\mathrm{q})$, where $\mathrm{P}(\mathrm{q})$ is the form factor. The gap between the q-ranges for the SLS and the available SANS data, comprised between $3 \times 10^{-3}$ and $10^{-2} \AA^{-1}$, is relatively important. Nevertheless, these experiments give us a good direct estimation of the chitosan persistence length that is 20 anyway determined at higher $\mathrm{q}$. The plot as a function of $\mathrm{q}$ exhibits three domains: i) a low-q Guinier regime; ii) an intermediate $\mathrm{q}^{-2}$ polymer coil regime, and finally iii) a $\mathrm{q}^{-1}$ domain at higher $\mathrm{q}$ characteristic of rigid rodlike behaviour for distances smaller than the persistence length $\mathrm{L}_{\mathrm{p}}$. This suite of variations can 25 be fitted satisfactorily to the form factor of a wormlike chain model with no excluded volume interactions ${ }^{68-72}$ (see ESI for details). This is justified by the small positive value of the second Virial coefficient $\mathrm{A}_{2}$ determined in the presence of a $0.2 \mathrm{M}$ excess of salt-see the Zimm plot in the inset of Fig. 1.

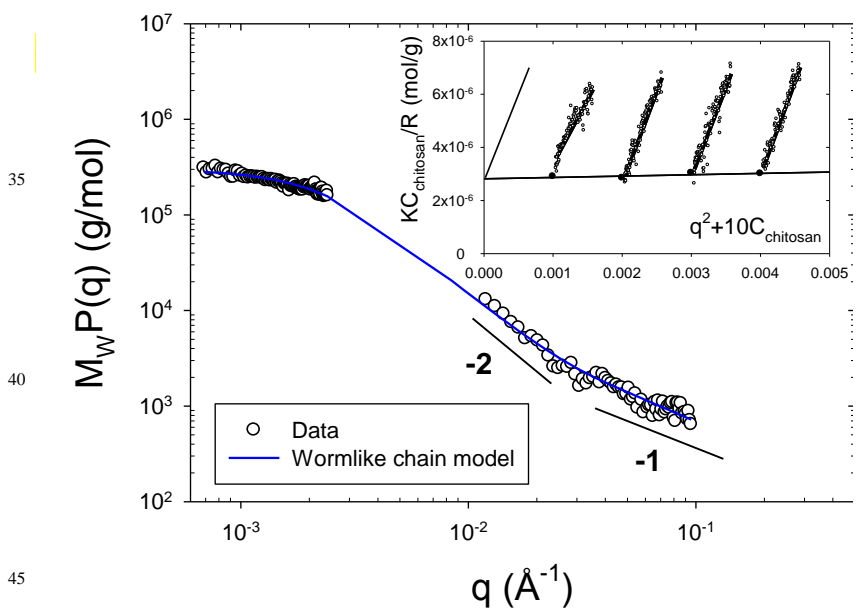

Fig. 1 Combined static light scattering and SANS spectra for a $0.1 \mathrm{~g} / \mathrm{l}$ chitosan solution. To superimpose both techniques we plot $\mathrm{M}_{\mathrm{W}} \mathrm{P}(\mathrm{q})$ as a function of q. The continuous line represents the fit of the data with a

50 wormlike chain model (see ESI). The Zimm plot of chitosan is presented in the inset.

The fit of the data yields $M_{W}=301 K$, in good agreement with the value determined using the Zimm analysis, the contour length ${ }_{55} \mathrm{~L}_{\mathrm{c}}=1116 \mathrm{~nm}$ corresponding to the theoretical value of a single strand chitosan chain and $L_{p}=9 \mathrm{~nm}$ : here $L_{p}$ is close to the intrinsic persistence length, the electrostatic additive contribution to the persistence length being negligible in the presence of $0.2 \mathrm{M}$ of salt. This value is close to that previously reported. ${ }^{55}$ It is ${ }_{60}$ determined directly by SANS for the first time. Therefore with chitosane and our SiNPs $(\mathrm{R} \sim 9 \mathrm{~nm})$ we are in the case $\mathrm{L}_{\mathrm{p}} / \mathrm{R} \sim 1$.

\section{Sequence of Phase Behaviours}

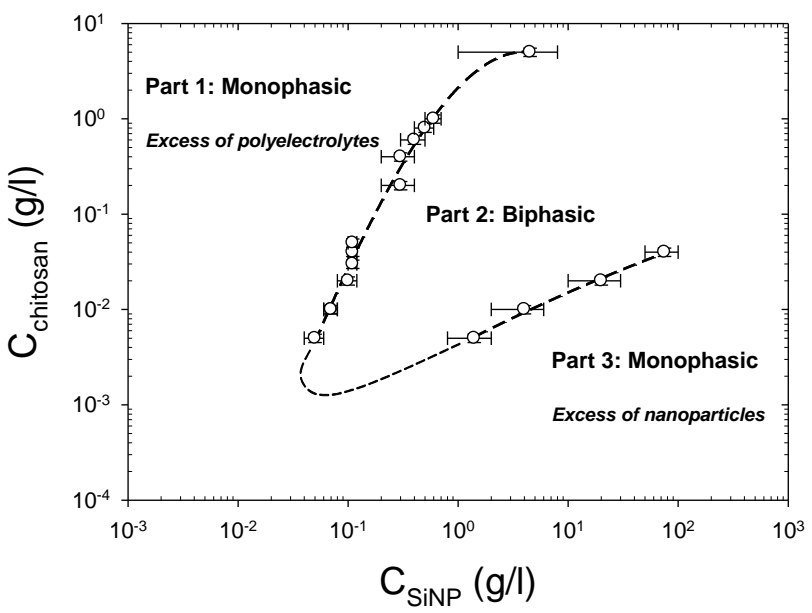

${ }_{65}$ Fig. 2 Sequence of phase behaviours in the chitosan concentration-SiNP concentration plane at $\mathrm{T}=20^{\circ} \mathrm{C}$ and fixed $\mathrm{pH} 4.5$.

This section deals with the phase behaviour and the SANS experiments performed on charged SiNPs in the presence of oppositely charged chitosan semiflexible chains at fixed 70 temperature $\mathrm{T}=20^{\circ} \mathrm{C}$. At the constant $\mathrm{pH}$ chosen (see above), 4.5, the silica particles undubiously carry a negative surface charge density (an estimate of the zeta potential from mobility -see ESIcorresponds to 25 negative effective charges).

In Figure 2, we have sketched the phase evolution of the 75 samples with the chitosan concentration (vertical axis) and the SiNPs concentration (horizontal axis) at equilibrium. For extremely low chitosan addition, the solutions are monophasic and transparent over the whole range of NPs concentration. When the chitosan concentration is increased, one observes for 80 intermediate NPs concentrations a phase separation: a dilute phase coexists with a more concentrated and viscous phase (see Part 2), while, at lower (Part 1) and higher (Part 3) NPs concentrations, the samples remain clear monophasic solutions.

Such shape of the phase diagram reported in Figure 2 is 85 reminiscent of "complex coacervation" ${ }^{1,52}$ due to electrostatic attraction between polyelectrolytes and oppositely charged NPs or proteins. ${ }^{38-41,45-49,73}$ The two kinds of objects can first associate in primary complexes, which then are neutral. Above a certain concentration they precipitate in rich and poor liquid phases, or 90 form fractal aggregates by controlled (diffusion limited or better reaction limited) aggregation, or solid clusters (this depends on the form of the attraction potential). ${ }^{50}$ Another aspect is the release of the counterions of both species, which has an entropic contribution on the free energy, as predicted and experimentally ${ }_{95}$ observed. ${ }^{53}$ In practice for our system, SANS results indicate that the concentrated lower phase contains the major part of the NPs 
and of the chitosan, while the upper fluid phase is a very dilute solution of both NPs and chitosan. It is noteworthy that the phase separation is observed for minute quantities of chitosan.

10

15

20

25

30

35
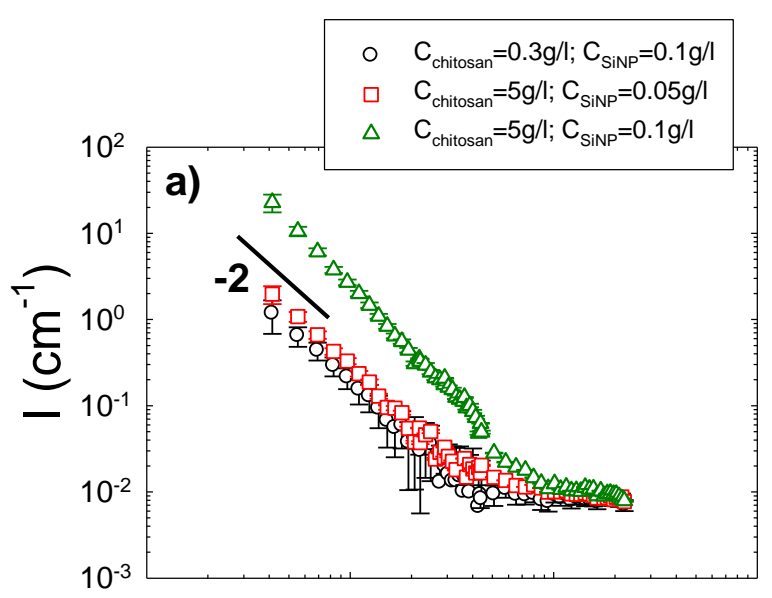

b)
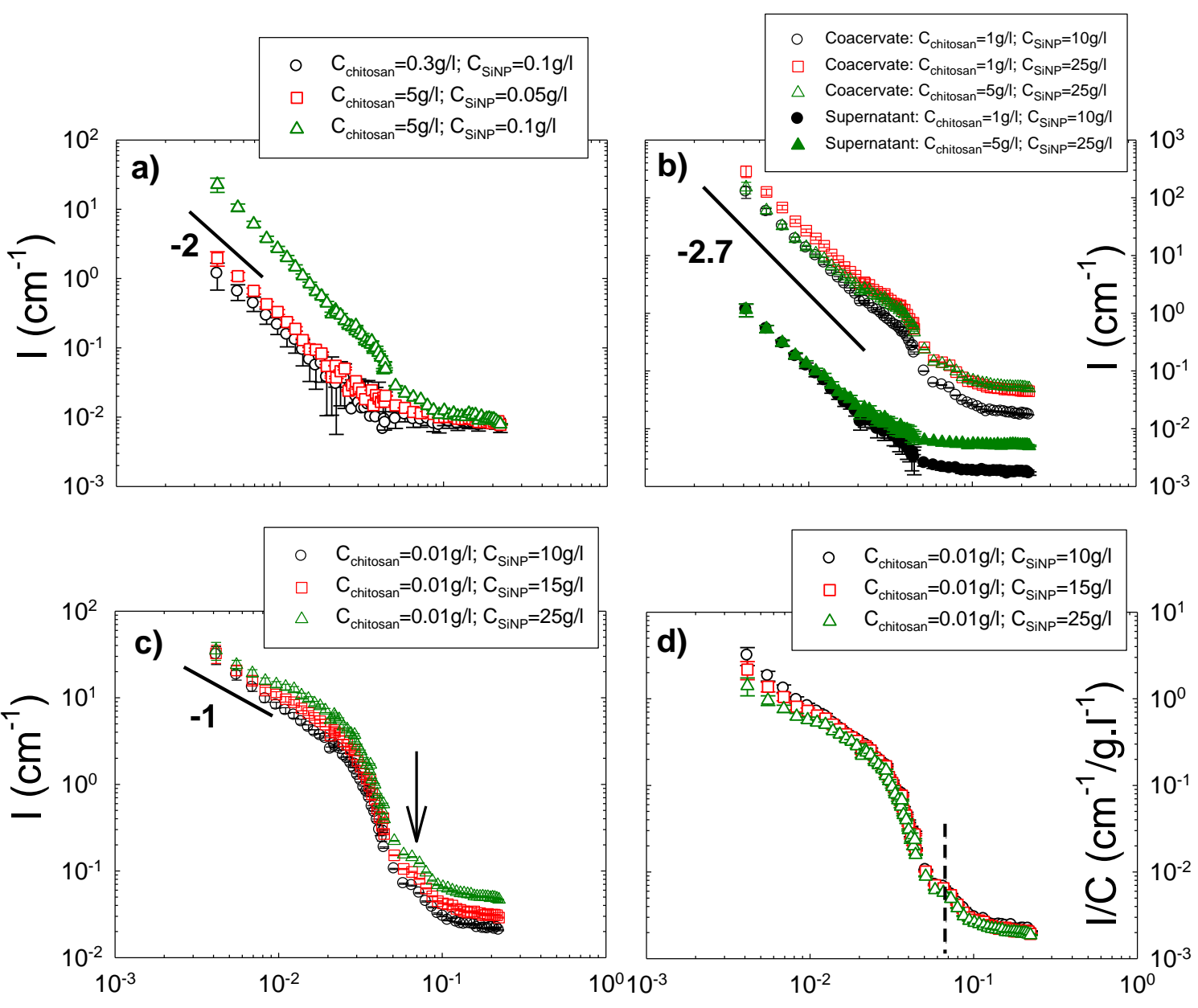

$q\left(\AA^{-1}\right)$

Fig. 3 Variation of the scattered intensity, I, with q obtained by SANS in chitosan/SiNP solutions in $\mathrm{D}_{2} \mathrm{O}$ at $\mathrm{pH}=4.5$ : (a) dilute $\mathrm{SiNP}$ solutions in the

90 presence of an excess of chitosan chains (monophasic domain \#1); (b) biphasic domain \#2 (for clarity the spectra corresponding to the supernatant phase have been shifted by one $\log$ unit); and (c) dilute chitosan solutions in the presence of an excess of SiNPs (monophasic domain \#3). (d) same as (c) but representation of the variation with q of 95 the ratio $\mathrm{I} / \mathrm{C}_{\mathrm{SiNP}}$. 
To gain insight into the arrangement of the SiNPs and the local structure of the complexes, SANS experiments were performed on solutions characterizing the different parts of the phase diagram: Part 1 (excess of chitosan chains-monophasic domain), ${ }_{5}$ Part 2 (Biphasic domain) and Part 3 (excess of SiNPSmonophasic domain). This is a powerful method for determining shapes of objects in solution over the range of 1-30 nm. Figure 3 shows the variations of the scattered intensity as a function of $q$ measured for each of the representative solutions in heavy water 10 and in the presence of $0.2 \mathrm{M} \mathrm{CH}_{3} \mathrm{COONa}$ and $0.3 \mathrm{M} \mathrm{CH}_{3} \mathrm{COOH}$ at $\mathrm{T}=20^{\circ} \mathrm{C}$. It is important to note that the SANS signal is dominated by the SiNPs, as expected from the high value of the SiNPs molecular volume (measurements in $\mathrm{D}_{2} \mathrm{O} / \mathrm{H}_{2} \mathrm{O}$ mixture eliminating the signal of chitosan by contrast matching, not 15 shown here, corroborate this estimate).

Part 1: Figure 3 a shows the SANS curves obtained for $\mathrm{C}_{\text {chitosan }}=0.3 \mathrm{~g} / \mathrm{l} / \mathrm{C}_{\mathrm{SiNP}}=0.1 ; \quad \mathrm{C}_{\text {chitosan }}=5 \mathrm{~g} / 1 / \mathrm{C}_{\mathrm{SiNP}}=0.05$ and $\mathrm{C}_{\text {chitosan }}=5 \mathrm{~g} / \mathrm{l} / \mathrm{C}_{\mathrm{SiNP}}=0.1 \mathrm{~g} / \mathrm{l}$ monophasic mixtures (dilute 20 solutions of SiNPs in the presence of an excess of chitosan). The scattering curve does not exhibit a Guinier regime with a plateau at low $\mathrm{q}$ associated to the finite size of the complexes but instead $\mathrm{a} \sim \mathrm{q}^{-2}$ behaviour in a rather extended $\mathrm{q}$ range, followed by a high $\mathrm{q}$ oscillation. This $\mathrm{q}^{-2}$ dependence suggests a Gaussian 25 distribution for the NPs inside the complexes, or branched quasifractal aggregates. The global size of the complexes being larger than $30 \mathrm{~nm}$.

Part 2: For this biphasic domain, the scattering varies as a $\mathrm{q}^{-\alpha}$ law with $\alpha$ ranging from 2.6 to 2.8 , characteristic of rather 30 compact fractal aggregates larger than $30 \mathrm{~nm}$ (the scattering curves do not exhibit a Guinier regime with a plateau at low q). This behavior is observed in the whole biphasic domain for dense phases as well as for supernatant phases, as seen in Figure $3 b$. The power law is followed at large q, as for part 1, by a high $\mathrm{q}$ 35 oscillation that will be discussed below.

Part 3: Figure 3c displays the scattering patterns for chitosan/SiNPs solutions at various SiNPs concentrations (Domain 3 of the phase diagram). The variations of the ratio $\mathrm{I} / \mathrm{C}_{\mathrm{SiNP}}$ of the scattered intensity over the SiNP concentration are 40 illustrated in Figure 3d. The most interesting feature of Figure 3d concerns the intermediate and high q-ranges where all the scattering curves superimpose on each other, thus indicating that the self-assemblies have the same structure at the spatial scale corresponding to these q-ranges. Furthermore in the lowest q45 range, the $q$ dependence of the scattered intensity tend to join for the lowest concentrations, a power law with an exponent close to -1 , which suggests a rod-like structure at the corresponding scale. This variation with $\mathrm{q}$ has never been observed in NPpolyelectrolyte complexes at our knowledge. The oscillation ${ }_{50}$ observed in the high q-range is the initial part of the oscillating term of the shape dependent form factor of the particle crosssection.

In the low q range, the shape of the scattering curves depends on the NPs concentration. The system with a very large excess of ${ }_{55} \mathrm{SiNPs}\left(\mathrm{C}_{\mathrm{SiNP}}=25 \mathrm{~g} / \mathrm{l}\right)$ tends to exhibit a Guinier regime with the beginning of a plateau in the low $\mathrm{q}$ range associated with the average finite size of the scattered objects (here a mixture of rigid-rods and of free SiNPs). This "plateau" is followed by a slight upturn probably associated to the presence of rods that 60 dominate the signal at very low q. For SiNPs concentrations of 10 $\mathrm{g} / \mathrm{l}$ and $15 \mathrm{~g} / \mathrm{l}$ the curves show neither a Guinier plateau nor a significant upturn of the scattered intensity in the low q-range: the characteristic $\mathrm{q}^{-1}$ rod dependence is measured down to the lowest $\mathrm{q}$, thus indicating that the rods dominating the SANS signal in ${ }_{65}$ this concentration domain are relatively long; i.e., larger than 30 $\mathrm{nm}$.

All curves of Figure 3 exhibit an oscillation occurring at the same value of $\mathrm{q}$ for all the systems investigated. However, the absence of a Guinier regime at low $\mathrm{q}$ and the contribution of the 70 incoherent background at high q prevented us from determining the global and cross-sectional dimensions of the nanorod aggregates with very high accuracy. Therefore, it was interesting to turn to SAXS experiments at the ESRF high brilliance light source.

\section{${ }_{75}$ Nanorods SAXS characterization}

Figure $4 \mathrm{a}$ shows the variations of the ratio $\mathrm{I} / \mathrm{C}_{\mathrm{SiNP}}$ of the scattered intensity over the SiNP concentration versus the scattering wave vector $\mathrm{q}$ for nanorod solutions at two different NPs concentrations, $\mathrm{C}_{\mathrm{SiNP}}=10 \mathrm{~g} / \mathrm{l}$ and $15 \mathrm{~g} / \mathrm{l}$. Due to the high mass and 80 concentration of the SiNPs, the SAXS signal is dominated by the scattering of the NPs; the signal of chitosan chains is negligible. The scattering pattern of free SiNPs is also represented in the high q range in Figure 4a. The scattering curves of the complexes exhibit the same overall behaviour for both SiNP concentrations, 85 characterized by the following sequence: a Guinier regime in the low $\mathrm{q}$ range associated with the finite size and mass of the scattered objects, one intermediate regime in which the $q$ dependence of the scattered intensity is described by a power law with an exponent close to -1 , an axial Guinier regime at higher $\mathrm{q}$ 90 corresponding to the size of the cross-section of the assemblies, and finally well-defined oscillations associated to the shapedependent form factor of the particle cross-section. The curve corresponding to the bare silica particles is also shown in Figure 4a. In the high q range, all the scattering curves (rod complexes 95 and bare silica NPs) superimpose on each other, thus indicating that rods have the same cross-section than that of free SiNPs.

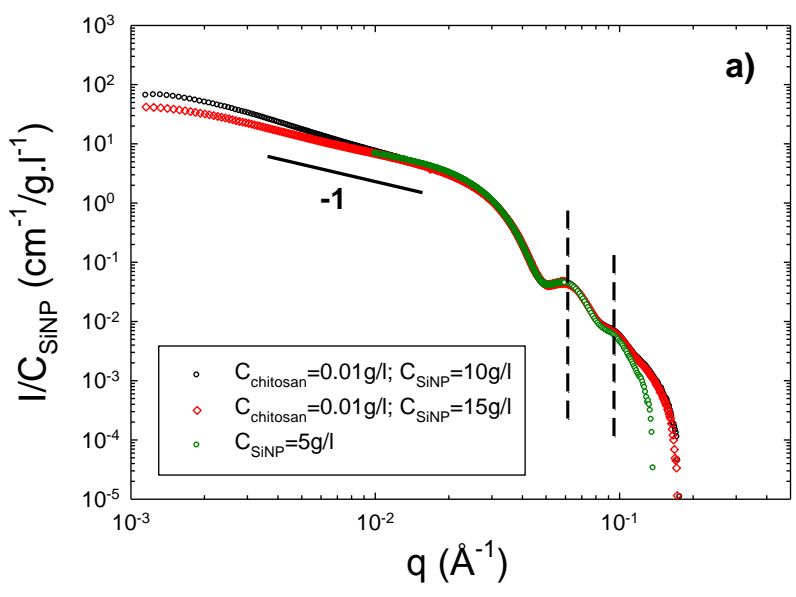


Fig. 4a Variation of the ratio $\mathrm{I} / \mathrm{C}_{\mathrm{SiNP}}$ with $\mathrm{q}$ obtained using SAXS experiments: comparison between free $\operatorname{SiNPs}\left(q>10^{-2} \AA^{-1}\right)$ and in the presence of $0.01 \mathrm{~g} / 1$ chitosan at $\mathrm{T}=20^{\circ} \mathrm{C}$.

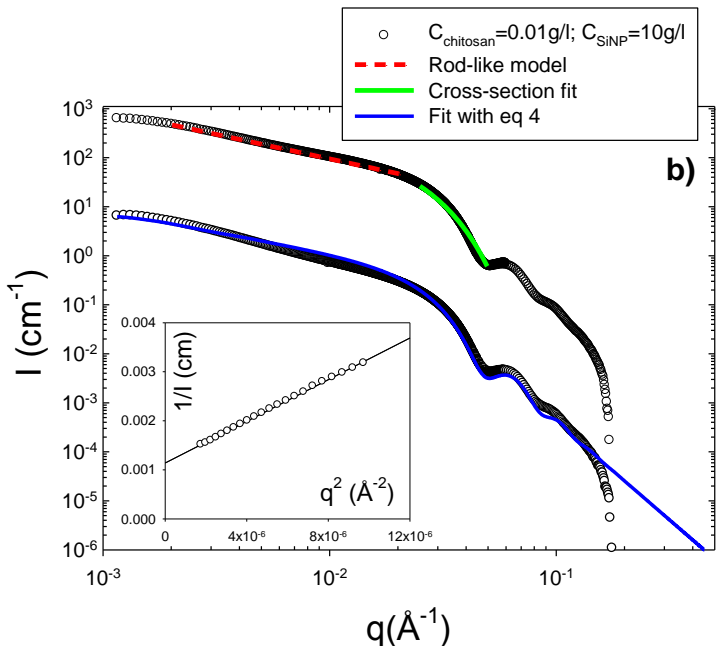

20 Fig. 4b SAXS spectra obtained for a $0.01 \mathrm{~g} / \mathrm{l}$ chitosan $/ 10 \mathrm{~g} / \mathrm{l} \mathrm{SiNP}$ solution in $0.2 \mathrm{M} \mathrm{CH}_{3} \mathrm{COONa}$ and $0.3 \mathrm{M} \mathrm{CH}_{3} \mathrm{COOH}$. Upper curve: the dashed curve represents the fit of the data in the intermediate regime with a rod-like model, and the solid curve represents the fit of the high q data by a Guinier expression for the form factor of the section (see ESI).

25 Bottom curve, for clarity the data have been shifted by two log units along the y-axis: the continuous solid line represents the best fit of the data obtained by a combination of spheres and cylinders form factors (see eq 4). The inset shows the plot of $1 / \mathrm{I}$ versus $\mathrm{q}^{2}$ and the best linear fit to the data.

\section{Inner conformation}

In the intermediate $\mathrm{q}$ regime, the scattering curves can be fitted satisfactorily by a rigid rod model. Figure $4 \mathrm{~b}$ shows the fit realized for a $0.01 \mathrm{~g} / \mathrm{l}$ chitosan $/ 10 \mathrm{~g} / \mathrm{l} \mathrm{SiNP}$ solution, that is in the very dilute regime for chitosan, by means of the form factor 35 derived for rigid rod particles, $\mathrm{P}(\mathrm{q})_{\mathrm{rod}}=\pi / \mathrm{qL}_{\mathrm{c}}$, where $\mathrm{L}_{\mathrm{c}}$ is the contour length. ${ }^{74}$ The high $\mathrm{q}$ data can be fitted by a Guinier expression for the form factor of the section (see ESI), giving the radius of gyration of the cross section, $r_{c}$. By fitting the two models above to the experimental data, after dividing by the 40 contrast as for individual particles, one can determine the mass per unit length of the rods, $\mathrm{M}_{\mathrm{L}}$, the section, $\mathrm{S}$, and the radius of gyration, $r_{c}$, of the cross section. From the fits of Figure $4 b$, we obtain $\mathrm{M}_{\mathrm{L}}=(98 \pm 9) \times 10^{3} \mathrm{~g} / \mathrm{mol} / \mathrm{nm}, \mathrm{S}=131 \pm 15 \mathrm{~nm}^{2}$, and $\mathrm{r}_{\mathrm{c}}=6 \pm 0.2$ $\mathrm{nm}$. If the rods consist of straight SiNPs monolayer wires, then 45 the mass per unit length can be calculated to be $\sim(\text { mass } / \text { diameter })_{\mathrm{SiNP}}=3 \times 10^{6} / 24=125 \times 10^{3} \mathrm{~g} / \mathrm{mol} / \mathrm{nm}, \quad$ a value slighly larger than the experimental determination. This discrepancy may be explained by the contribution of free NPs to the SAXS signal (see next part).

${ }_{50}$ The radius of gyration of the cross-section of such a nanorod is given by $r_{c}{ }^{2}=R^{2} / 2$. Using the experimental value of $r_{c}=6 \mathrm{~nm}$, one obtains $\mathrm{R}=8.5 \mathrm{~nm}$, which is a value in good agreement with the experimental one determined for bare silica particles (see Figure SI-6 in the ESI). In all cases, the determined local structural 55 parameters are consistent with a well-ordered single-strand 1D SiNP self-assembly whose cross-section is that of a single SiNP. This is corroborated by the oscillations observed at higher $q$ that are identical for all the samples for $\mathrm{q}>4 \times 10^{-2} \AA^{-1}$ (when $\mathrm{S}(\mathrm{q}$ ) 1) and that are reasonably well reproduced with $\mathrm{P}(\mathrm{q})$ calculated for ${ }_{60}$ the spherical SiNPs with a radius R of $9.2 \mathrm{~nm}$ (see Figure 4 and ESI ).

\section{Overall shape of the nanorods}

The low q data have been fitted by the classical Guinier 65 expression, $1 / I(q)=1 / I(0) \times\left(1+q^{2} R_{G}^{2} / 3\right)$, which provides the average radius of gyration, $\mathrm{R}_{\mathrm{G}}$, and the zero-wave vector intensity, $\mathrm{I}(0)$, associated to the weight-average molecular weight, $\mathrm{M}_{\mathrm{W}}$, of the rods. For rodlike particles with large aspect ratio $\left(\mathrm{L}_{\mathrm{c}}<<\mathrm{L}_{\mathrm{p}}\right), \mathrm{R}^{2}{ }_{\mathrm{G}}=\mathrm{L}^{2} / 12$, where $\mathrm{L}$ is the average contour length of 70 the rod. For $\mathrm{C}_{\mathrm{SiNP}}=10 \mathrm{~g} / \mathrm{l}$, we obtain $\mathrm{R}_{\mathrm{G}}=77 \mathrm{~nm}, \mathrm{~L}=267 \mathrm{~nm}$ and $\mathrm{M}_{\mathrm{W}}=(31 \pm 3) \times 10^{6} \mathrm{~g} / \mathrm{mol}$. Inspection of the data calls for two remarks. First, the objects formed behave as rigid rods, as shown by the comparison between the calculated value $L=12^{1 / 2} R_{G}=267$ $\mathrm{nm}$ and the experimental value of ${ }_{75} \mathrm{M}_{\mathrm{W}} / \mathrm{M}_{\mathrm{L}}=\left(31 \times 10^{6}\right) /\left(98 \times 10^{3}\right)=316 \mathrm{~nm}\left(\mathrm{M}_{\mathrm{L}}\right.$ is the silica mass per unit length of the rodlike object). Secondly, the average number of SiNPs constituting a nanorod is, neglecting the Virial effects, $\sim \mathrm{M}_{\mathrm{W} \text {,nanorod }} / \mathrm{M}_{\mathrm{W}, \mathrm{SiNP}} \sim 11$ (or $\sim 14-15$ if one considers the ratio between the rod length and the NPs diameter, equal to 267/18.4).

80 For $\mathrm{C}_{\text {chitosan }}=0.01 \mathrm{~g} / \mathrm{l} / \mathrm{C}_{\mathrm{SiNP}}=15 \mathrm{~g} / \mathrm{l}$ solutions, the zero-wave vector scattered intensity, $\mathrm{I}(0)$, is slightly lower due to the enhanced contribution to the signal of the free SiNPs, and thus showing that the proportion of both populations has to be taken into account: free SiNPs and rodlike self-assemblies (see part 85 3.4.). The low-q signal is, however, still dominated by the rodlike behaviour.

\section{Polyelectrolyte Concentration Influence on the Shape of the NPs Self-Assemblies}

To gain insight into the shape of the NPs self-assemblies, 90 complementary cryo-TEM experiments were performed on solutions characterizing the three parts of the phase diagram, using also cryo-TEM as a support for the discussion.

\section{Part 1, excess of chitosan:}

95 Evidence in favour of dilute randomly self-assembled NPs structures of $300-400 \mathrm{~nm}$, as already suggested by the $\mathrm{q}^{-2}$ dependence observed in the SANS spectra was provided by cryoTEM images of solutions containing an excess of chitosan $(5 \mathrm{~g} / \mathrm{l})$ and $0.1 \mathrm{~g} / \mathrm{l} \mathrm{SiNPs}$ (Figures 5a). A common observation in these 100 micrographs is that NPs do not form single-strand structures but instead form flexible branched structures with variable lengths, cross-sections, and aggregation numbers (mostly between 20 and 50). Thus an apparent fractal dimension of 2 is in agreement with such branched or contorted structure. Also, no free NPs are 105 observed. Thus, assuming that all chitosan chains are involved in the self-assemblies, we can estimate that the number of chains per complex is around $16 \sim$ (Number of chitosan chains)/(Number of NPs $\times<\mathrm{N}_{\mathrm{agg}}>$ ), with $\left\langle\mathrm{N}_{\mathrm{agg}}>\sim 30\right.$ the average NPs aggregation number.

110

Part 2, biphasic domain:

Figures 5b show micrographs of vitrified coacervate phases (concentrated viscous phases). Here, we see dense and globular SiNP aggregates larger than $500 \mathrm{~nm}$ composed of most of the 
NPs and chitosan chains. These observations corroborate the SANS analysis.

\section{Part 3, excess of SiNPs:}

5 Imaging of chitosan/SiNP solutions using Cryo-TEM corroborates the SANS and SAXS results indicating the presence of straight rodlike structures in monophasic solution with large excess of NPs. Figure 5c show representative images that clearly demonstrate the presence of aligned nanorod-like objects. Some 10 objects (see right hand picture of Figure 5c) present some kinks probably due to a collapse of the rod structure due to ice melting under beam irradiation. Thus for the structure of the objects in bulk solution we better rely on the SAXS, which definitely shows rodlike behaviour. All the rods exhibit uniform diameters of 15 approximately $20 \mathrm{~nm}$, which are in agreement with the diameters calculated from $r_{c}$ values determined from SAXS data (assuming a circular cross-section). Beyond agreement with SANS/SAXS, TEM gives new information on the rods lengths, which are variable (mostly between 160 and $280 \mathrm{~nm}$ ). In the light of 20 invariant diameters, and in the absence of branched structures, the observed nanorods are thought to correspond to single strand rigid NP assemblies as already shown by the scattering analysis and the experimental value of the linear mass density.

The low electronic contrast of the biopolymer does not enable 25 to identify the number of chain per complex and their arrangement in the nanorods structure. Higher resolution, Cs corrected and energy filtered TEM would be necessary to reveal that information. To shed some light on this aspect, we have estimated the amount of chitosan per complexes from SAXS 30 measurements. This simple calculation have been made on the basis of the average number of individual nanoparticles and of the nanorods determined by fitting the scattering pattern by the following equation: ${ }^{75}$

$$
I(q)=I_{\text {rods }}(q)+I_{\text {spheres }}(q)
$$

35 where $I_{\text {rods }}(q)$ and $I_{\text {spheres }}(q)$ are the scattered intensities related respectively to the form factor of the rods and of the spheres (see ESI for equations). For the rods, we used the classical form factor derived for cylinders (see ESI). The best agreement with the data presented in Figure $4 \mathrm{~b}$ is obtained for $\phi_{\text {spheres }}=4.3 \times 10^{-3}$, $40 \phi_{\text {rods }}=2.3 \times 10^{-4}, \quad R_{\text {spheres }}=R_{\text {cylinders }}=8.5 \mathrm{~nm}, \quad \sigma=0.11$, and for $\mathrm{L}_{\text {cylinders }}=250 \mathrm{~nm}$ (see bottom curve of Figure $4 \mathrm{~b}$, fit realized with eqs 2, 3 and 4 using the SASfit program ${ }^{76}$ ). Then, considering that SiNPs are in excess and that, according to cryo-TEM, only SiNPs present in complexes display the ribbed texture attributed 45 to chitosan chain binding, one can hypothesize that all the chitosan chains are involved in the complexes. Finally, one derives an average concentration of 1.8 chains of chitosan per nanorods. This result first shows that very few chains are needed to allow SiNPs organisation into nanorods. A second striking 50 point is that the average contour length of the biopolymers ( 943 $\mathrm{nm})$ is significantly higher than the average nanorods length $(\sim 250 \mathrm{~nm})$ suggesting that chitosan chains are somehow wrapped around SiNPs. This could be permitted by adequacy between the NPs surface curvature and the chitosan chains bending, controlled 55 by its persistence length. This is possible since $\mathrm{L}_{\mathrm{p}}(7.5 \mathrm{~nm}) \sim \mathrm{R}$ $(8.5 \mathrm{~nm})$. The number of NPs is $250 \mathrm{~nm} /(8.5 \times 2)=14.7$, corresponding to a chain length of $62 \mathrm{~nm}$ per NPs, i.e. slightly more than one NP circumference $(53 \mathrm{~nm})$.

This suggests that the chain wrapping around the SiNP is 60 somehow helical, and thus adds rigidity explaining the single strand rod-like structure. This is in striking contrast with DNA packaging into chromatin, where an almost rigid polymer with bare persistence length $\mathrm{L}_{\mathrm{p}}$ about $50 \mathrm{~nm}$ is compacted around small oppositely charged histones with $\mathrm{R} \approx 3.5 \mathrm{~nm} .{ }^{77}$ Here a 65 much more flexible polymer (intrinsic persistence length $\mathrm{L}_{\mathrm{p}} \approx 7.5$ $\mathrm{nm})$ can be rigidified, only via electrostatic interactions, into rodlike structure by nanoparticles with radius such as $\mathrm{L}_{\mathrm{p}} / \mathrm{R} \approx 1$.

The average length of these $1 \mathrm{D}$ assemblies $(250 \mathrm{~nm})$ gives a lower bound for the nanorods persistence length, $\mathrm{L}_{\mathrm{p}, \text { rod,min }}$, and 70 can thus be used to estimate the Young modulus, E, of such rods. Indeed, in the case of a rigid and uniform $\operatorname{rod} E=4 B_{s} / \pi R^{4}$, where $\mathrm{B}_{\mathrm{s}}$ is the bending stiffness and $\mathrm{R}$ the radius of the section. ${ }^{78,79} \mathrm{~A}$ lower bound for the modulus of such nanorods can be estimated: $\mathrm{E}_{\min }=\left(\mathrm{L}_{\mathrm{p}, \text { rod,min }} \times \mathrm{kT} \times 4\right) /\left(\pi \mathrm{R}^{4}\right)=1.79 \times 10^{5} \mathrm{~Pa}$.

75 
$s$

10

a)

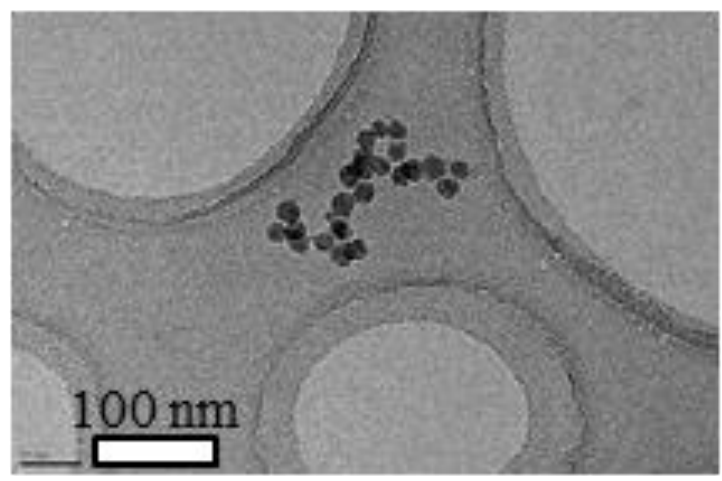

15

20

30

35

b)

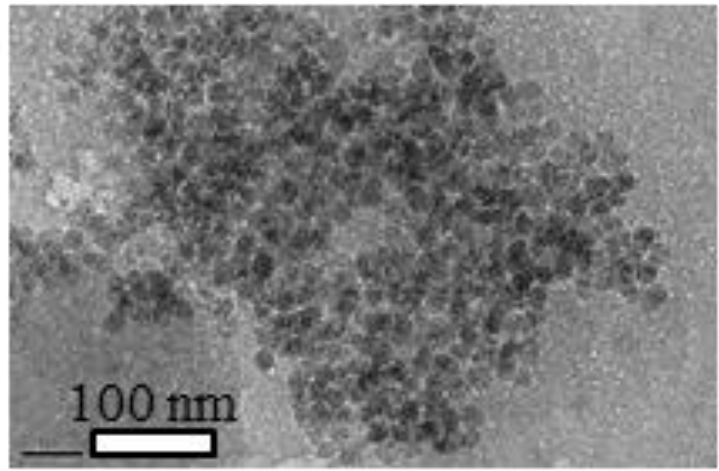

c)

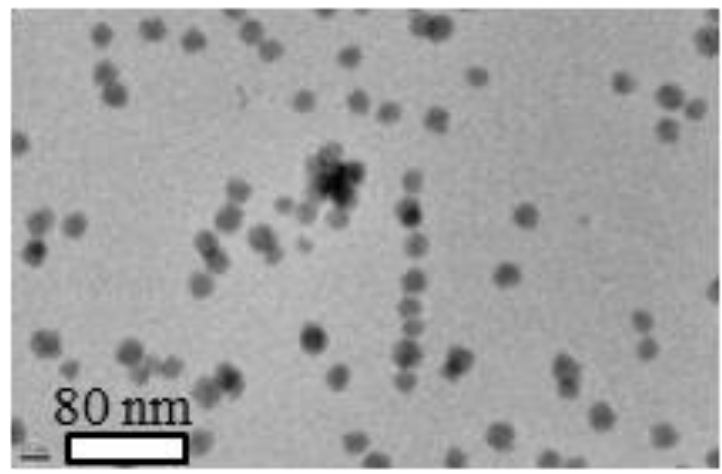

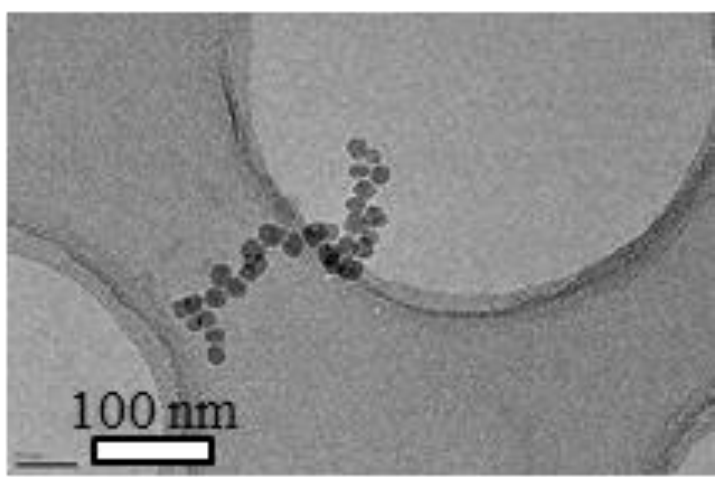
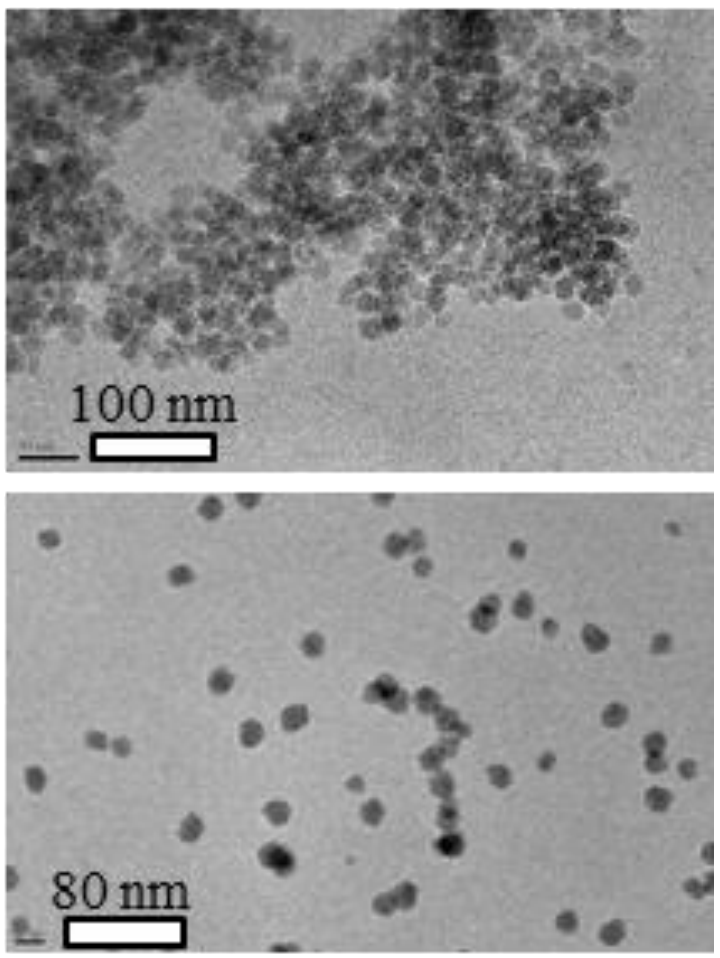

Fig. 5 Cryo-TEM images of a) a vitrified $C_{\text {chitosan }}=5 \mathrm{~g} / 1 / \mathrm{C}_{\mathrm{SiNP}}=0.1 \mathrm{~g} / \mathrm{l}$ monophasic solution (Part 1); b) a coacervate phase with initial concentrations of $\mathrm{C}_{\text {chitosan }}=1 \mathrm{~g} / \mathrm{l}$ and $\mathrm{C}_{\mathrm{SiNP}}=10 \mathrm{~g} / \mathrm{l}$ (Part 2); and c) a $\mathrm{C}_{\text {chitosan }}=0.01 \mathrm{~g} / \mathrm{l} / \mathrm{C}_{\mathrm{SiNP}}=10 \mathrm{~g} / \mathrm{l}$ monophasic solution $($ Part 3 ) in the presence 50 of $0.3 \mathrm{M} \mathrm{CH}_{3} \mathrm{COOH}$ and $0.2 \mathrm{M} \mathrm{CH}_{3} \mathrm{COONa}$. 


\section{Influence of the ratio $L_{p} / R$}

As seen in the previous part, it is possible to obtain well-defined 1D rodlike self-assemblies of SiNPs in the presence of an excess 5 of NPs. This suggests a role of the polyelectrolyte rigidity, and the importance of using less rigid PELs. Therefore the polyelectrolyte poly-L-lysine (PLL), displaying -like chitosanepositive charges along its flexible backbone, was chosen; the second partner, $10 \mathrm{~nm}$ SiNPs, remained unchanged. The 10 concentration of $\mathrm{KBr}$ was fixed to $0.2 \mathrm{M}$ (PLL being purchased with $\mathrm{Br}^{-}$counterions, excess salt $\mathrm{KBr}$ was chosen) in order to keep the same ionic strength than in the chitosan-SiNPs systems in the presence of $0.2 \mathrm{M} \mathrm{CH}_{3} \mathrm{COONa}$. Within these experimental conditions, $\mathrm{L}_{\mathrm{p}}(\mathrm{PLL}) \sim \mathrm{nm}$ (PLL intrinsic persistence length), and ${ }_{15} \mathrm{~L}_{\mathrm{p}} / \mathrm{R}$, is close to 0.1 .

As for the chitosan-SiNPs system, the mixed solutions are monophasic and transparent in the presence of an excess of PLL (domain 1), or of SiNPs (domain 3). A biphasic (domain 2) is observed in the intermediate range of concentrations (with 20 boundaries pretty close to those determined previously with chitosan), where one rich phase of white coacervate coexists with an upper dilute and limpid phase. To investigate the effect of, $\mathrm{L}_{\mathrm{p}} / \mathrm{R}$ on the structure of the complexes and on the formation of nanorods, SANS experiments were performed on two PLL/SiNPs 25 solutions in the presence of an excess of NPs (domain 3). The ratio between the concentration of PLL and SiNPs is identical for both samples and is the same as that used for characterizing the complexes of SiNPs with semi-rigid chitosan in the same domain \#3. Both representative samples appear visually as a unique phase

30 but are opalescent and were stable on an observation time scale of several months.

The scattering patterns obtained for $\mathrm{C}_{\mathrm{PLL}}=0.01 \mathrm{~g} / 1 /$ $\mathrm{C}_{\mathrm{SiNP}}=10$, and $\mathrm{C}_{\mathrm{PLL}}=0.001 \mathrm{~g} / \mathrm{l} / \mathrm{C}_{\mathrm{SiNP}}=1$ and showed in Figure 6 do not exhibit any Guinier regime with a plateau at low $\mathrm{q}$ 35 associated to the finite size of the complexes but instead a $\mathrm{q}^{-2}$ regime behavior in an extended $\mathrm{q}$ range, followed by the first oscillation associated to the form factor of the cross-section of the $10 \mathrm{~nm}$ radius SiNPs. The variations of the ratio $\mathrm{I} / \mathrm{C}_{\mathrm{SiNP}}$ of the scattered intensity over SiNP concentration are illustrated in ${ }_{40}$ Figure 6b. A first mostly interesting feature is that the scattering curves superimpose on each other in the whole q-range indicating that the complexes, which are larger than $30 \mathrm{~nm}$, have the same structure. This suggests that the latter depends only on $L_{p} / R$ in this domain. A second is the low $\mathrm{q}$ slope $\left(\mathrm{q}^{-2}\right.$ dependence $)$ 45 suggesting either a Gaussian distribution for the SiNPs inside the complexes, or branched aggregates. We have here an important result, because it profoundly differs from that obtained with the semiflexible polyelectrolyte chitosan (which showed a $\mathrm{q}^{-1}$ law due to the formation of nanorods within the same experimental 50 conditions). This suggests the major role played by the polyelectrolyte persistence length. Here, PLL, a flexible polyelectrolyte, with $\mathrm{L}_{\mathrm{p}} / \mathrm{R} \sim 0.1$, does not induce the formation of $1 \mathrm{D}$ structures.

55
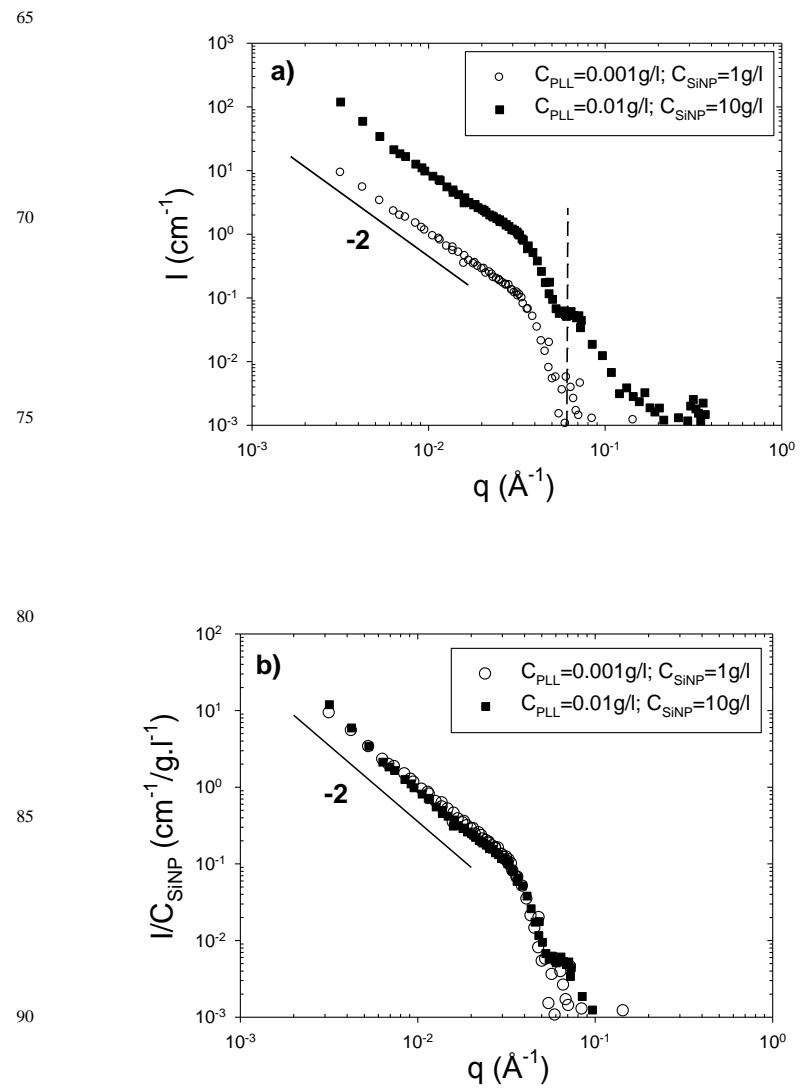

Fig. 6 (a) SANS spectra obtained for Poly-L-lysine (PLL)/SiNP solutions in domain \#3 (excess of NPs) in the presence of $0.2 \mathrm{M} \mathrm{KBr}$. (b) same as 95 (a) but representation of the variation with $\mathrm{q}$ of the ratio $\mathrm{I} / \mathrm{C}_{\mathrm{SiNP}}$.

The results, summarized in the "general phase diagram" (see Figure 7), point for the first time the role of the characteristic ratio $L_{p} / R$ in the control of the structure, making plausible a 100 cognitive generalization to many other candidate species.

105 110

115

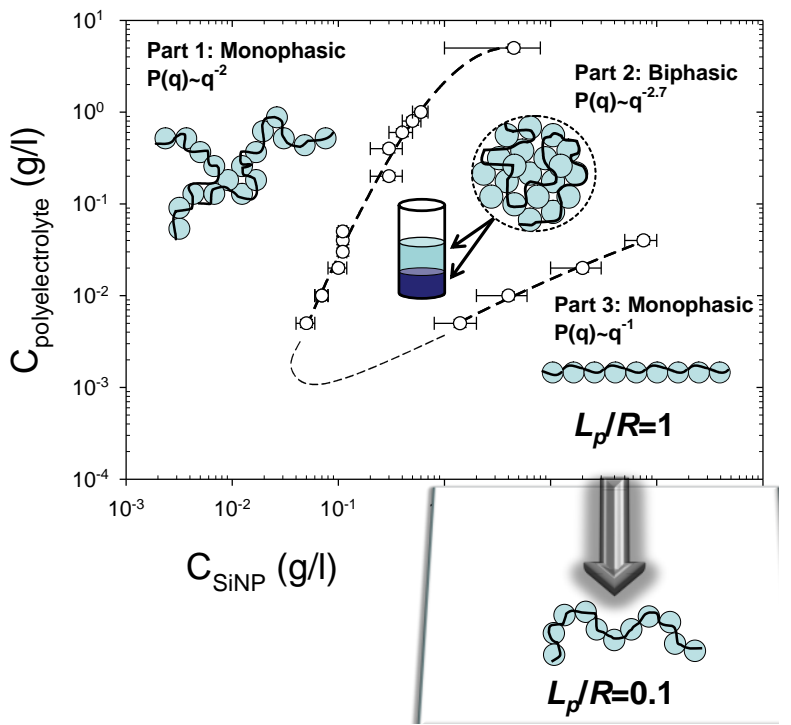


Fig. 7 Sequence of phase behaviours in the Chitosane polyelectrolyte concentration - SiNP concentration plane in the presence of an excess of salt. The form factor, $\mathrm{P}(\mathrm{q})$, scales as $\sim \mathrm{q}^{-}$ ${ }^{2}, \sim \mathrm{q}^{-2.7}$, and $\mathrm{q}^{-1}$ respectively in part 1,2 , and 3 of the phase 5 diagram for $\mathrm{L}_{\mathrm{p}} / \mathrm{R} \sim 1$. A flexible polyelectrolyte (PLL), with $\mathrm{L}_{\mathrm{p}} / \mathrm{R}=0.1$, does not induce the formation of nanorods in domain \#3.

\section{Conclusions}

This work can be commented from different points of view. First, 10 it can be seen as an exploration of the different structures obtained in the phase diagram resulting from an associative mechanism, here involving electrostatic complexation, and in given concentrations range, leading to phase separation, the socalled "complex coacervation". The specificities of three kinds of 15 structure are clearly cut thanks to the combination of SANS, SAXS, and cryo-TEM for the three phase diagram regions. From these structures in the three regions of the phase diagram, we can try to imagine the mechanisms for a system of chains and particles with possibly strong interactions since all particles are 20 multiply charged and all chain segments are charged.

The obtained shapes are summarized in Figure 7. When chains are in excess, branched objects are formed made of linear short strands, while when NPs are in excess linear objects appear. In the stoichiometric range, which corresponds to the biphasic 25 regime, more quasi-3d compact objects are formed. They are observed both in the supernatant and in the coacervate (dense) phase (the fractal dimension determined by SANS is the same in both phases). This suggests that the dense objects are formed prior to phase separation. Their shape would be induced by the 30 high number of particles per monomer in the stoichiometric complexes, while more expanded shapes correspond to an insufficient number of NPs (part 1) or to an insufficient number of chains (part 3). In parts 1 and 3 as well as in the supernatant phase (part 2), it is very likely that individual complexes are 35 formed. From this first point of view, it seems that the shapes of the complexes are dictated by the monomer/NP ratio.

Second, the succession of different NPs organisations is not observed with such consistency in most of the former works, and in particular, rodlike shapes as well defined as here - as seen at 40 the light of SAXS measurements- are rarely observed. Our result is due first to the use of a semiflexible polyelectrolyte, and second to a good adequacy between persistence length and NPs radius. Thus a second important ratio appears to be $\mathrm{L}_{\mathrm{p}} / \mathrm{R}$. Indeed, these results show the major role played by the polyelectrolyte 45 persistence length. In particular, the flexible polyelectrolyte which we have used, poly-L-lysine, with $\mathrm{L}_{\mathrm{p}} / \mathrm{R} \sim 0.1$, does not induce the formation of $1 \mathrm{D}$ structures. We point here that the ratio $\mathrm{L}_{\mathrm{p}} / \mathrm{R}$ plays a pivotal role in the formation of $1 \mathrm{D}$ structures.

Finally the structure of the chain itself may play a role: the 50 chitosan chain possesses gentle helical shape, which can help a lot to make the structure more rigid. The helix could wrap gently the NPs. This rejoins some pictures and evaluation formerly proposed by theorists. ${ }^{38}$ However, while this was proposed for the interaction between one chain and one NP, this is observed here ${ }_{55}$ in the case of complexes involving several NPs per chain, where the structure formed renders easy a precise check. The use of a natural polyelectrolyte polysaccharide involving such helical structure appears to be a good choice, although the chain anchorages at the surface of the NPs are not as specific as they 60 could be for proteins. ${ }^{80,81}$

\section{Acknowledgments}

This work was supported by a doctoral fellowship from the CNRS and the CEA (L. S.). The authors thank the Laboratoire Léon Brillouin (LLB, CEA Saclay, France), the Institut Laue${ }_{65}$ Langevin (ILL, Grenoble, France), and the European Synchrotron Radiation Facility (ESRF, Grenoble) for beam time allocations. We wish to thank J. Gummel and I. Grillo for their help during the SAXS and the SANS experiments, respectively.

\section{Notes and references}

$70{ }^{a}$ Laboratoire Matière et Systèmes Complexes (MSC), UMR CNRS 7057, Université Paris Diderot-Paris 7, Bâtiment Condorcet, 10 rue Alice Domon et Léonie Duquet, 75205 Paris cedex 13, France. E-mail : eric.buhler@univ-paris-diderot.fr, Fax : 3315727 6211, Tel : 3315727 6139

$75{ }^{b}$ Laboratoire Léon Brillouin, UMR 12 CEA-CNRS, CEA Saclay, 91191 Gif-sur-Yvette, France.

${ }^{c}$ Laboratoire Chimie de la Matière Condensée de Paris, UMR 7574, UPMC, Collège de France, 11 place Marcelin Berthelot, 75005 Paris, France.

80

$\dagger$ Electronic supplementary information (ESI) available: Scattering methods, single solute solutions characterization, and models. See DOI: $10.1039 / \ldots \ldots$

85

1. R. Bhattacharya, C. Ranjan Patra, S. Wang, L. Lu, M. J. Yaszemski, D. Mukhopadhyay, P. Mukherjee, Adv. Func. Mater., 2006, 16, 395 400.

2. P. L. Anelli, N. Spencer, J. F. Stoddart, J. Am. Chem. Soc., 1991, 113, 5131.

3. J. Otsuki, M. Tsujino, T. Iizaki, K. Araki, M. Seno, K. Takatera, T. Watanabe, J. Am. Chem. Soc., 1997, 119, 7895.

4. A. P. de Silva, H. Q. N. Gunaratne, C. P. McCoy, J. Am. Chem. Soc., 1997, 119, 7891.

95 5. M. Grzelczak, J. Vermant, E. M. Furst, L. M. Liz-Marza, ACS Nano, 2010, 4, 3591-3605.

6. Z. Nie, A. Petukhova, E. Kumacheva, Nat. Nanotechnol., 2010, 5, 15.

7. S. C. Glotzer, M. A. Horsch, C. R. Iacovella, Z. Zhang, E. R. Chan, X. Zhang, Curr. Opin. Colloid Interface Sci., 2005, 10, 287.

100 8. S. C. Glotzer, M. J. Solomon, Nat. Mater., 2007, 6, 557.

9. G. A. DeVries, M. Brunnbauer, Y. Hu, A. M. Jackson, B. Long, B. T. Neltner, O. Uzun, B. H. Wunsch, F. Stellacci, Science, 2007, 315, 358.

10. W. U. Huynh, J. J. Dittmer, A. P. Alivisatos, Science, 2002, 295, 1052425.

11. M. Rycenga, J. M. McLellan, Y. Xia, Adv. Mater., 2008, 20, 2416.

12. Z. Tang, N. A. Kotov, M. Giersing, Science, 2002, 297, 237.

13. A. Courty, A. Mermet, P. A. Albouy, E. Duval, M. P. Pileni, Nat. Mater., 2005, 4, 395.

110 14. D. Zerrouki, J. Baudry, D. Pine, P. Chaikin, J. Bibette, Nature, 2008, 455, 380.

15. Z. Nie, D. Fava, E. Kumacheva, S. Zou, G. C. Walker, M. Rubinstein, Nat. Mater., 2007, 6, 609.

16. S. A. Maier, P. G. Kik, H. A. Atwater, S. Meltzer, E. Harel, B. E. 115 Koel, A. A. G. Requicha, Nat. Mater., 2003, 2, 229.

17. S. A. Maier, M. L. Brongersma, P. G. Kik, S. Meltzer, A. A. G. Requicha, H. A. Atwater, Adv. Mater., 2001, 13, 1501.

18. W. Nomura, M. Ohtsu, T. Yatsui, Appl. Phys. Lett., 2005, 86, 181108.

19. C.-J. Wang, L. Huang, B. A. Parviz, L. Y. Lin, Nano Lett., 2006, 6 , $120 \quad 2549$. 
20. T. Yatsui, Y. Ryu, T. Morishima, W. Nomura, T. Kawazoe, T. Yonezouva, M. Washizu, H. Fujita, M. Ohtsu, Appl. Phys. Lett., 2010, 96, 133106.

21. J. N. Anker, W. Paige Hall, O. Lyandres, N. C. Shah, J. Zhao, R. P. Van Duyne, Nat. Mater., 2008, 7, 442.

22. G. Kawamura, Y. Yang, M. Nogami, Appl.Phys. Lett., 2007, 90, 261908.

23. T. C. Deivaraj, N. L. Lala, J. Y. Lee, J. Colloid and Interface Sci., 2005, 289, 402.

10 24. V. M. Cepak, C. R. Martin, J. Phys. Chem. B, 1998, 102, 9985.

25. M. H. Huang, A. Choudrey, P. Yang, Chem. Comm., 2000, 12, 1063.

26. C. J. Murphy, N. R. Jana, Adv. Mater., 2002, 14, 80.

27. N. Toshima, Y. Wang, Chem. Lett., 1993, 1611.

28. M. Cao, Y. Wang, C. Guo, Y. Qi, C. Hu, E. Wang, J. Nanosci. Nanotech., 2004, 4, 824.

29. B. Wiley, Y. Sun, B. Mayers, Y. Xia, Chem. Eur. J., 2005, 11, 454.

30. T. Harada, H. Fujiwara, Journal of Physics: Conference Series, 2007, 61, 394-398.

31. M. E. Leunissen, R. Dreyfus, F. C. Cheong, D. G. Grier, R. Sha, N. C.

20 Seeman, P. M. Chaikin, Nat. Mater., 2009, 8, 590.

32. K.K. Caswell, J. N. Wilson, U. H. F. Bunz, C. J. Murphy, J. Am. Chem. Soc., 2003, 125, 13914.

33. K. D. Hermanson, S. O. Lumsdon, J. P. Williams, E. W. Kaler, O. D. Velev, Science, 2001, 294, 1082.

25 34. C. Ribeiro, E. J. H. Lee, E. Longo, E. R. Leite, ChemPhysChem, 2006, 7, 664.

35. S. Shanbhag, Z. Tang, N. A. Kotov, ACS Nano, 2007, 1, 126.

36. K. Liu, Z. Nie, N. Zhao, W. Li, M. Rubinstein, E. Kumacheva, Science, 2010, 329, 197-200.

30 37. Z. Nie, D. Fava, M. A. Winnik, M. Rubinstein, E. Kumacheva, J. Am. Chem. Soc., 2008, 130, 3683.

38. C. Holm, J. F. Joanny, K. Kremer, R. R. Netz, P. Reineker, T. Vilgis, R. Winkler, Adv. Polym. Sci., 2004, 166, 67.

39. F. Cousin, J. Gummel, D. Ung, F. Boué, Langmuir, 2005, 21, 9675.

35 40. Jonghoon Lee, Yuri O. Popov, and Glenn H. Fredrickson, J. Chem. Phys., 2008, 128, 224908.

41. G. Nizri, A. Makarsky, S. Magdassi and Y. Talmon, Langmuir, 2009, 25, 1980-1985.

42. A. Y. Grosberg, T. T. Nguyen, B. I. Shklovskii, Rev. Mod. Phys., $40 \quad 2002, \mathbf{7 4}, 329$.

43. T. T. Nguyen and B. I. Shklovskii, J. Chem. Phys., 2001, 114, 13.

44. C. Y. Kong and M. Muthukumar, J. Chem. Phys., 1998, 109, 15221527.

45. M. Jonsson, P. Linse, J. Chem. Phys., 2001, 115, 10975.

45 46. K. Keren, Y. Soen, G. Ben Yoseph, R. Gilad, E. Braun, U. Sivan, Y. Talmon, Phys. Rev. Lett., 2002, 89, 088103.

47. (a) J. Gummel, F. Boué, D. Clemens, F. Cousin, Soft Matter, 2008, 4, 1653-1664. (b) J. Gummel, F. Boué, B. Demé, F. Cousin, Journal of Physical Chemistry B, 2006, 110 (49), 24837 - 24846.

50 48. C. L. Cooper, A. Goulding, A. Basak Kayitmazer, S. Ulrich, S. Stoll, S. Turksen, S. Yusa, A. Kumar, P. L. Dubin, Biomacromolecules, 2006, 7, 1025-1035.

49. A. Basak Kayitmazer, S. P. Strand, C. Tribet, W. Jaeger, P. L. Dubin, Biomacromolecules, 2007, 8, 3569.

55 50. F. Cousin, J. Gummel, S. Combet, F. Boué, Adv. Col. Int. Sci., 2011, 167, 71-84.

51. C. G. De Kruif, F. Weinbreck, R. De Vries, Curr. Opin. Coll. Interface Sci, 2004, 9, 340.

52. H. G. Bungenberg de Jong, H. R. Kruyt, Proc. K. Ned. Akad. Wet., $1929,32,849$.

53. J. Gummel, F. Cousin, F. Boué, J. Am. Chem. Soc., 2007, 129, 58065807.

54. B. Bharti, J. Meissner, G. H. Findenegg, Langmuir, 2011, 27, $9823-$ 9833.

65 55. E. Buhler, O. Guetta, M. Rinaudo, Int. J. Polym. Anal. and Charac., $2000,6,155$.

56. E. Buhler, M. Rinaudo, Macromolecules, 2000, 33, 2098-2106.

57. C. Esquenet, E. Buhler, Macromolecules, 2001, 34, 5287.

58. C. Esquenet, P. Terech, F. Boué, E. Buhler, Langmuir, 2004, 20, $70 \quad 3583-3592$.

59. M. Rasmusson, S. Wall, Colloid Surf. A, 1997, 122, 169-181.
60. P. Van der Meeren, H. Saveyn, S. Bogale Kassa, W. Doyen, R. Leysen, Phys. Chem. Chem. Phys., 2004, 6, 1408-1412 .

61. D.F. Evans, H. Wennerström, The Colloidal Domain; Wiley-VCH: NewYork, 1994.

62. J. Appell, G. Porte, E. Buhler, J. Phys. Chem. B, 2005, 109, 1318613194.

63. K. S. Schmitz, An Introduction to Dynamic Light Scattering by Macromolecules; Academic Press: London, 1990.

80 64. C. Esquenet, E. Buhler, Macromolecules, 2002, 35, 3708-3716.

65. D. E. Koppel, J. Chem. Phys., 1972, 57, 4814.

66. S. W. Provencher, Makromol. Chem., 1985, 82, 632.

67. R. Ravindra, K. R. Krowidi, A. A. Kahn, Carbohydrate Polymers, 1998, 36, 121-127.

85 68. J. S. Pedersen, P. Schurtenberger, Macromolecules, 1996, 29, 7602.

69. W. Burchard, K. Kajiwara, Proc. R. Soc. London, Ser. A, 1970, 316, 185.

70. P. Sharp, V. A. Bloomfield, Biopolymers, 1968, 6, 1201.

71. E. Buhler, F. Boué, Eur. Phys. J. E, 2003, 10, 89.

90 72. E. Buhler, F. Boué, Macromolecules, 2004, 37, 1600-1610.

73. (a) E. Buhler, J. Appell, G. Porte, J. Phys. Chem. B, 2006, 110, 6415-

6422. (b) F. Carn, N. Steunou, M. Djabourov, T. Coradin, F. Ribot, J.

Livage, Soft Matter, 2008, 4, 735-738. (c) F. Carn, O. Durupthy, B.

Fayolle, T. Coradin, G. Mosser, M. Schmutz, J. Maquet, J. Livage, N.

${ }_{95}$ Steunou, Chem. Mater., 2010, 22, 398-408. (d) F. Carn, F. Boué, M.

Djabourov, N. Steunou, T. Coradin, J. Livage, S. Floquet, E. Cadot, E.

Buhler, Soft Matter 2012, 8, 2930-2944.

74. (a) J. des Cloizeaux, Macromolecules, 1973, 6, 403. (b) S. Ulrich, E. Buhler, J.-M. Lehn, New J. Chem., 2009, 33, 271-292.

100 75. N. Jouault, R. Nguyen, M. Rawiso, N. Giuseppone, E. Buhler, Soft Matter 2011, 7, 4787.

76. SASfit at: http://kur.web.psi.ch/

77. A. A. Zinchenko, K. Yoshikawa, D. Baigl, Phys. Rev. Lett., 2005, 95, 228101.

105 78. L. Shi, F. Carn, F. Boué, E. Buhler, ACS Macro Lett., 2012, 1, 857861.

79. K. S. Bloom, Chromosoma, 2008, 117, 103.

80. I. Morfin, E. Buhler, F. Cousin, I. Grillo, F. Boué, Biomacromolecules, 2011, 12, 859.

110 81. Y. Ruff, E. Buhler, S. J. Candau, E. Kesselman, Y. Talmon, J. M. Lehn, J. Am. Chem. Soc., 2010, 132, 2573-2584. 